\section{CardioRenal Medicine}

\title{
The Effect of Mitochondrial Complex I-Linked Respiration by Isoflurane Is Independent of Mitochondrial Nitric Oxide Production
}

\author{
Fuqi Xu ${ }^{a}$ Shigang Qiao ${ }^{a, b}$ Hua Li ${ }^{a}$ Yanjun Deng ${ }^{a}$ Chen Wang ${ }^{a, b}$ \\ Jianzhong $A n^{b}$ \\ ${ }^{a}$ Department of Anesthesiology and Perioperative Medicine, Suzhou, China; ${ }^{b}$ Institute of \\ Clinical Medicine Research, Suzhou Hospital (West District) Affiliated to Nanjing Medical \\ University, Suzhou Science and Technology Town Hospital, Suzhou, China
}

\section{Keywords}

Isoflurane $\cdot$ Mitochondria $\cdot$ Complex I $\cdot$ Respiration $\cdot$ Nitric oxide

\begin{abstract}
Background: Anesthetic preconditioning (APC) of the myocardium is mediated in part by reversible alteration of mitochondrial function. Nitric oxide (NO) inhibits mitochondrial respiration and may mediate APC-induced cardioprotection. In this study, the effects of isoflurane on different states of mitochondrial respiration during the oxidation of complex I-linked substrates and the role of NO were investigated. Methods: Mitochondria were isolated from Sprague-Dawley rat hearts. Respiration rates were measured polarographically at $28^{\circ} \mathrm{C}$ with a computer-controlled Clark-type $\mathrm{O}_{2}$ electrode in the mitochondria $(0.5 \mathrm{mg} / \mathrm{mL})$ with complex I substrates glutamate/malate $(5 \mathrm{mM})$. Isoflurane $(0.25 \mathrm{mM})$ was administered before or after adenosine diphosphate (ADP)-initiated state 3 respiration. The NO synthase (NOS) inhibitor L-N $\mathrm{N}^{5}$-(1-iminoethyl)-ornithine (L-NIO, $\left.10 \mu \mathrm{M}\right)$ and the NO donor S-nitroso-N-acetylpenicillamine (SNAP, $1 \mu \mathrm{M}$ ) were added before or after the addition of ADP. Results: Isoflurane administered in state 2 increased state 2 respiration and decreased state 3 respiration. This attenuation of state 3 respiration by isoflurane was similar when it was given during state 3. L-NIO did not alter mitochondrial respiration or the effect of isoflurane. SNAP only, added in state
\end{abstract}

Fuqi Xu and Shigang Qiao contributed equally to this work. 
Xu et al.: Is NO Involved in the Effect of Mitochondrial Complex I-Linked Respiration by Isoflurane?

3 , decreased state 3 respiration and enhanced the isoflurane-induced attenuation of state 3 respiration. Conclusion: Isoflurane has clearly distinguishable effects on different states of mitochondrial respiration during the oxidation of complex I substrates. The uncoupling effect during state 2 respiration and the attenuation of state 3 respiration may contribute to the mechanism of APC-induced cardioprotection. These effects of isoflurane do not depend on endogenous mitochondrial NO, as the NOS inhibitor L-NIO did not alter the effects of isoflurane on mitochondrial respiration.

(C) 2018 The Author(s)

Published by S. Karger AG, Basel

\section{Introduction}

Anesthetic preconditioning (APC) is effective as ischemic preconditioning in protecting the heart against ischemia-reperfusion injury by decreasing myocardial infarct size and improving postischemic functional recovery [1-3]. Although the precise mechanisms responsible for APC-induced cardioprotection remain incompletely characterized, the reversible alteration of mitochondrial function initiated by volatile anesthetics has been implicated in cardioprotection [4]. The effects of volatile anesthetics on mitochondrial respiration and the electron transport chain (ETC) has been demonstrated in recent studies [5-7]. Ljubkovic et al. [6] showed that APC-treated heart mitochondria exhibited a better preservation of respiration and adenosine triphosphate (ATP) synthesis after hypoxia stress than non-APC treated mitochondria did. Hanley et al. [8] found that halothane, isoflurane, and sevoflurane dosedependently inhibited NADH:ubiquinone oxidoreductase (complex I) in pig heart submitochondrial particles. These studies showed that volatile anesthetics might directly affect mitochondria complexes and reactive oxygen species (ROS) production to modulate mitochondrial respiration and bioenergetics.

Volatile anesthetics may trigger preconditioning, in part, by inducing the formation of ROS and reactive nitrogen species and by the release of nitric oxide (NO) $[9,10]$. NO exerts a number of actions that are beneficial during myocardial ischemia-reperfusion $[10,11]$. For example, it may act as a trigger and mediator of isoflurane-induced delayed preconditioning in rabbit myocardium [12]. Furthermore, isoflurane potentiates ischemic postconditioning via an NO-dependent mechanism [13]. It has been suggested that mitochondria contain NO synthase (NOS) and produce NO to regulate respiration [14]. NO inhibits mitochondrial respiration by binding to respiratory chain enzymes, and modulates mitochondrial ATP-dependent $\mathrm{K}^{+}$channels [15]. Riess et al. [5] reported that, in isolated heart mitochondrial preparation, sevoflurane administered in state 2 attenuated state 3 respiration. The attenuation was mediated by ROS but not by mitochondrial $\mathrm{K}_{\mathrm{ATP}}$ channel opening. Therefore, the interaction between NO and the ETC may be important in APC-induced cardiac protection.

Because mitochondrial $\mathrm{O}_{2}$ consumption is different in each respiratory state, it is important to understand the effects of volatile anesthetics on the different states of mitochondrial respiration. The difference in $\mathrm{O}_{2}$ consumption in each state is tightly linked to the regulation of tissue $\mathrm{O}_{2}$ levels [16]. In addition, the respiration rate of mitochondria is one of the main factors for the regulation of mitochondrial ROS generation $[17,18]$. To date, the role of volatile anesthetics on different states of mitochondrial respiration (i.e., states 2-4) has not been addressed in detail. It is also unclear whether NO, like mitochondrial ROS, is involved in the effect of isoflurane on mitochondrial respiration. This study was designed to explore: (1) the effect of isoflurane on different mitochondrial respiration states during the oxidation of complex I-linked substrates, and (2) whether mitochondrial NO is involved in the effect of isoflurane on different mitochondrial respiratory states. 
Xu et al.: Is NO Involved in the Effect of Mitochondrial Complex I-Linked Respiration by Isoflurane?

Fig. 1. Experimental protocols used to measure mitochondrial respiration. State 2 respiration was initiated by the addition of 5 mm glutamate and malate; state 3 respiration was initiated by the addition of $250 \mu \mathrm{M}$ ADP. a Isoflurane $(0.25 \mathrm{mM})$ was added in state 2 or state 3 after applying the complex I substrates glutamate and malate. $\mathbf{b}$ The NOS inhibitor L-NIO or the NO donor SNAP was added in state 1,2 , or 3 with or without isoflurane. CTL, control group; ISO, isoflurane; GM, glutamate and malate; Mito, mitochondria; ADP, adenosine diphosphate.

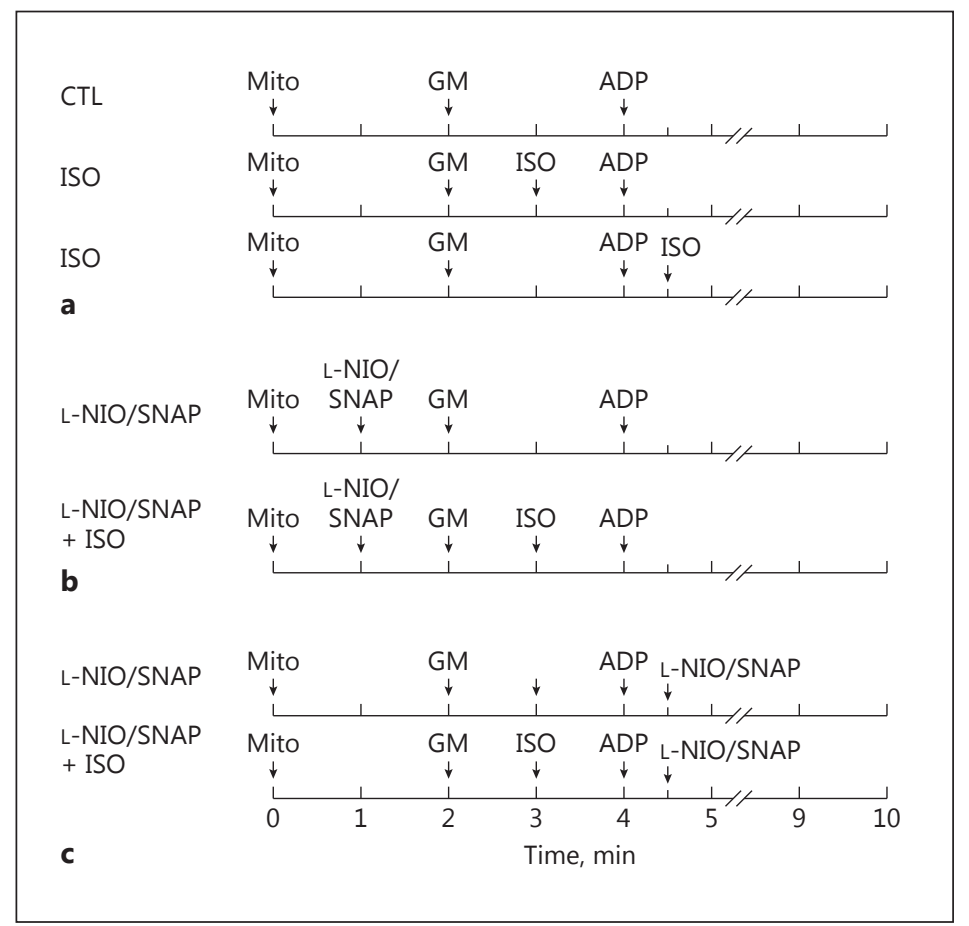

\section{Materials and Methods}

\section{Isolation of Mitochondria}

Male SD rats at 8-10 weeks of age maintained on a standard diet were used. Animals used in this study were approved by the Suzhou Science and Technology Town Hospital Institutional Animal Care and Use Committee. They were anesthetized with pentobarbital sodium $(50 \mathrm{mg} / \mathrm{kg}$ i.p.) and following decapitation, the hearts were quickly excised and plunged into an ice-cold isolation buffer containing (in mM) mannitol 200, sucrose 50, $\mathrm{KH}_{2} \mathrm{PO}_{4}$ 5, EGTA 1, 3-(N-morpholino)propanesulfonic acid (MOPS) 5, and 0.1\% bovine serum albumin ( $\mathrm{pH} 7.3$, adjusted with potassium hydroxide). Large vessels and auricles were discarded and remaining blood was removed. The ventricles were minced into $1-\mathrm{mm}^{3}$ pieces. The isolation of mitochondria was performed at ${ }^{\circ} \mathrm{O} \mathrm{C}$ as described previously [6]. The tissue was homogenized 4 times for $15 \mathrm{~s}$ each time with $30 \mathrm{~s}$ intervals. The homogenate was centrifuged at $800 \mathrm{~g}$ for $10 \mathrm{~min}$ and the obtained pellet was rehomogenized and recentrifuged. The resulting supernatants were centrifuged at $6,000 \mathrm{~g}$ for $10 \mathrm{~min}$, the pellets resuspended in $25 \mathrm{~mL}$ isolation buffer, and the suspension was then centrifuged at $800 \mathrm{~g}$ for another $10 \mathrm{~min}$. The supernatant containing the mitochondrial fraction was further centrifuged at 8,000 $\mathrm{g}$ for 10 min. This final pellet was resuspended in $1 \mathrm{~mL}$ isolation buffer without EGTA and kept on ice. The total protein concentration was determined by bicinchonicic acid protein assay with bovine serum albumin as a standard.

\section{Measurement of Mitochondrial Oxygen Consumption}

Mitochondrial respiration was measured polarographically at $28^{\circ} \mathrm{C}$ with a computer-controlled Clarktype $\mathrm{O}_{2}$ electrode (Hansatech Instruments Ltd, Norfolk, UK) in $0.5 \mathrm{~mL}$ respiratory buffer containing (in mM): $\mathrm{KCl} 130, \mathrm{~K}_{2} \mathrm{PH}_{4}$ 5, MOPS 20, EGTA 2.5, $\mathrm{Na}_{4} \mathrm{P}_{2} \mathrm{O} 70.001$ EDTA, and 0.1\% BSA (pH 7.2 adjusted with potassium hydroxide). Mitochondrial suspension $(0.5 \mathrm{mg} / \mathrm{mL})$ were added in respiratory buffer in the presence of complex I substrate glutamate (5 $\mathrm{mm}$ ) plus malate (5 $\mathrm{mm})$.

\section{Experimental Protocols}

To study the actions of isoflurane and NO on mitochondrial respiration, isoflurane $(0.25 \mathrm{mM})$, the NO donor S-nitroso-N-acetylpenicillamine (SNAP, $1 \mu \mathrm{M}$ ), or the NOS inhibitor L-N ${ }^{5}$-(1-iminoethyl)-ornithine (LNIO, $10 \mu \mathrm{M})$ were added according to the protocols in Figure 1. Glutamate (5 mM) and malate (5 mM) were 
Xu et al.: Is NO Involved in the Effect of Mitochondrial Complex I-Linked Respiration by Isoflurane?

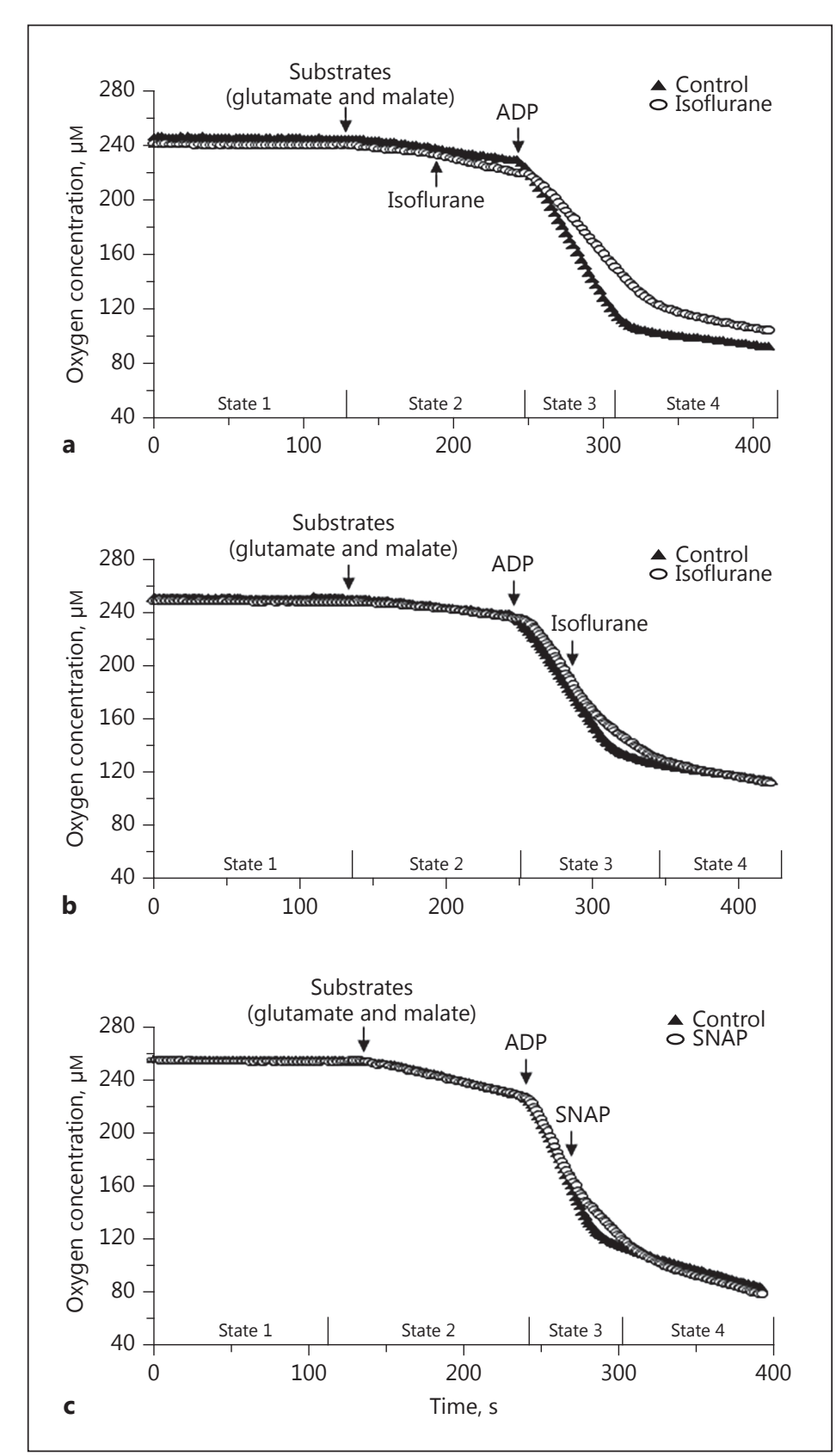

Fig. 2. Representative recording traces of mitochondrial respiration. Isoflurane $(0.25 \mathrm{~mm})$ was added in state 2 (a) or state 3 (b). c The NO donor, SNAP $(1 \mu \mathrm{M})$ was added in state 3. ADP, adenosine diphosphate.

added at $2 \mathrm{~min}$ and adenosine diphosphate (ADP, $250 \mu \mathrm{M}$ ) was added at $4 \mathrm{~min}$. Isoflurane was added at $3 \mathrm{~min}$ or after ADP was administered (Fig. 1a). L-NIO or SNAP was added before substrates were administered (1 min, Fig. 1b) or after ADP-initiated state 3 respiration (4.5 min, Fig. 1c).

These experiments used the conventional definitions for mitochondrial respiration states. State 2 is the state of $\mathrm{O}_{2}$ consumption in the presence of substrates without added ADP. State 3 is the state of maximal $\mathrm{O}_{2}$ consumption after the addition of ADP (ADP phosphorylation). State 4 is the state of minimal $\mathrm{O}_{2}$ consumption after the added ADP is depleted. The representative traces of respiration are shown in Figure 2. The traces indicate a high functional quality and structural integrity of mitochondria after the isolation procedure, as shown by the respiratory control ratio (RCR, state 3/state 4 ) of $9.8 \pm 1.5$ with the substrates glutamate and malate. 
Xu et al.: Is NO Involved in the Effect of Mitochondrial Complex I-Linked Respiration by Isoflurane?

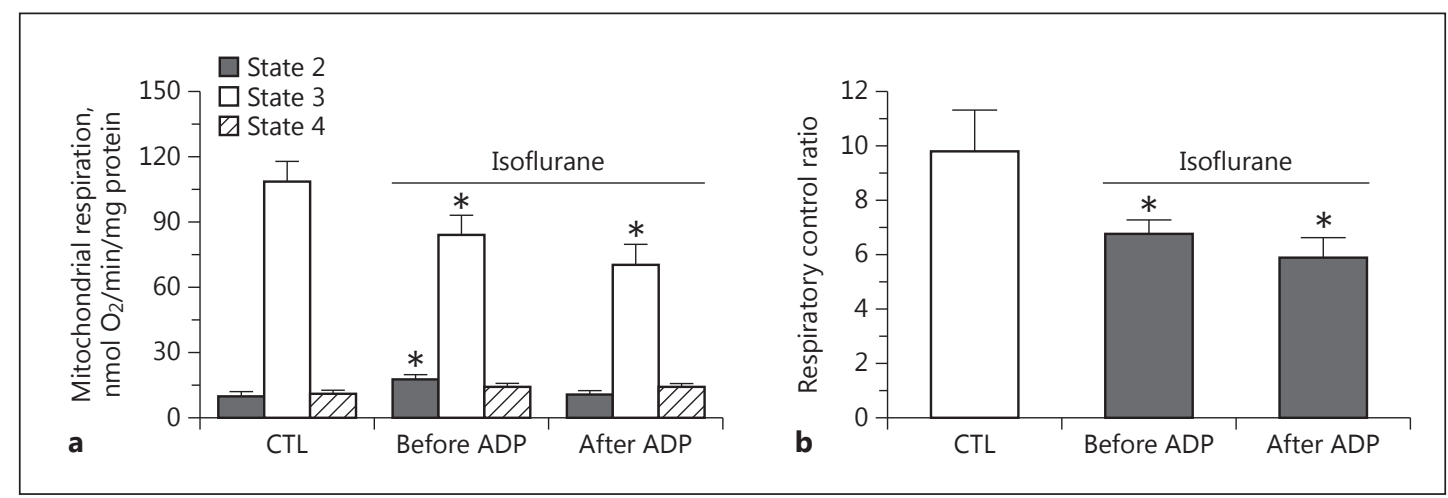

Fig. 3. Summarized data of the effects of isoflurane $(0.25 \mathrm{~mm})$ on mitochondrial respiration (states 2-4; a) and respiratory control ratio (b) when isoflurane was added, before or after ADP initiated state 3 respiration in the presence of the complex I substrates glutamate and malate. ${ }^{*} p<0.05$ compared to control group. ${ }^{\#} p<0.05$ compared to the group in which isoflurane was given in state 2 . CTL, control group; ADP, adenosine diphosphate.

Drugs and Chemicals

Isoflurane was purchased from Abbot Laboratories (Abbott Park, IL, USA). L-NIO was obtained from Calbiochem (San Diego, CA, USA). ADP, glutamate, malate, SNAP, and protease were obtained from Sigma (St. Louis, MO, USA). Isoflurane $(10 \mu \mathrm{L})$ stock solution was made by putting $10 \mu \mathrm{L}$ of anesthetic in $1 \mathrm{~mL}$ respiration buffer, and then sonicating for $10 \mathrm{~min} ; 5 \mu \mathrm{L}$ of this stock solution was added into the chamber containing $500 \mu \mathrm{L}$ of mitochondrial suspension. The concentration of isoflurane was analyzed by gas chromatography (Schimadzu, Kyoto, Japan). The average concentration of isoflurane was $0.25 \pm 0.02 \mathrm{mM}$. L-NIO was dissolved in respiration buffer at $1 \mathrm{mM}$ and SNAP was dissolved in DMSO at $100 \mathrm{mM}$, and they were then diluted to 100 $\mu \mathrm{M}$ by respiration buffer as a stock solution before the experiment. The stock solution of L-NIO or SNAP was added to $500 \mu \mathrm{L}$ mitochondrial suspension.

\section{Statistical Analysis}

All data are expressed as means \pm SD. A two-way analysis of variance was used to assess the overall difference between groups. The Student Newman Keuls' multiple-comparison post hoc test was used to differentiate within-group differences. Differences between means were considered significant when $p<$ 0.05 (two-tailed).

\section{Results}

\section{Effect of Isoflurane on State 2 and State 3 Respiration}

In this protocol, isoflurane administered in state 2 increased the state 2 respiration rate and significantly attenuated state 3 respiration compared to the control group (Fig. 3a). Consequently, the RCR was lower in the isoflurane group than in the control group (Fig. 3b). To observe the direct effect of isoflurane on state 3 respiration, the anesthetic was applied shortly after ADP was added, and isoflurane also attenuated the state 3 respiration (Fig. 3a). In this case, the RCR was also lower than in the control group.

\section{Role of NOS Inhibitor L-NIO in the Effect of Isoflurane}

To study the role of NO in the effect of isoflurane on mitochondrial respiration, the NOS inhibitor L-NIO was added before the administration of substrates and isoflurane (Fig. 1b) or after ADP application in state 3 (Fig. 1c). L-NIO did not alter state 3 respiration and did not affect the isoflurane-induced increase in state 2 respiration or decrease in state 3 respiration 
Xu et al.: Is NO Involved in the Effect of Mitochondrial Complex I-Linked Respiration by Isoflurane?

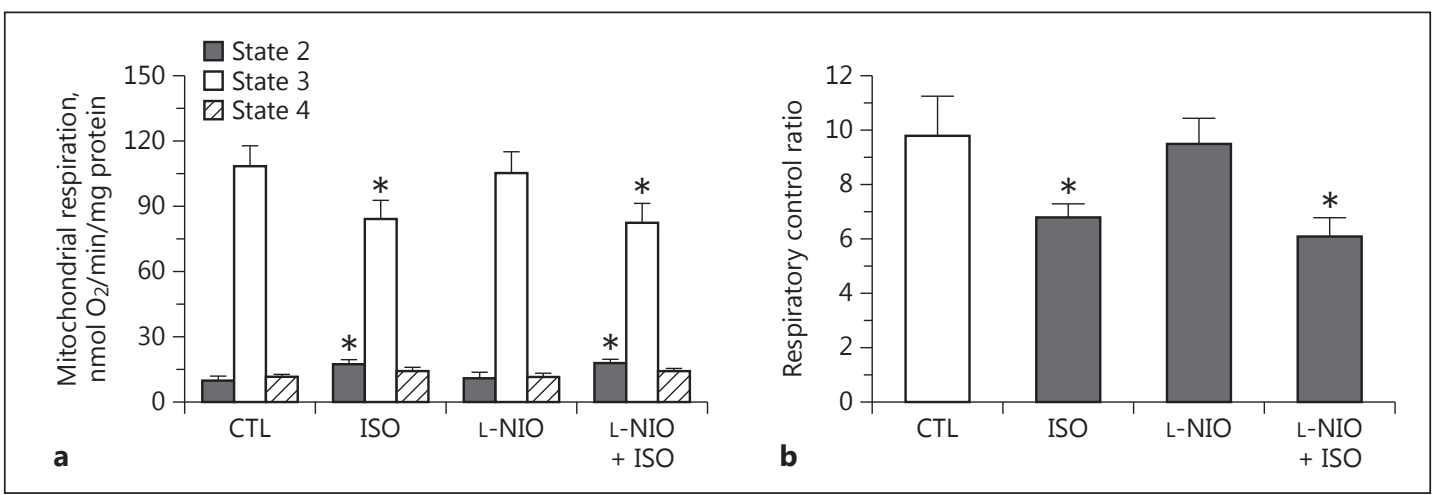

Fig. 4. Summarized data of the effects of the NOS inhibitor L-NIO (10 $\mu \mathrm{M}$, applied in state 1) on mitochondrial respiration (states 2-4; a) and respiratory control ratio (b) with or without isoflurane (applied in state 2 ) in the presence of the complex I substrates glutamate and malate. ${ }^{*} p<0.05$ compared to control group. CTL, control group; ISO, isoflurane.

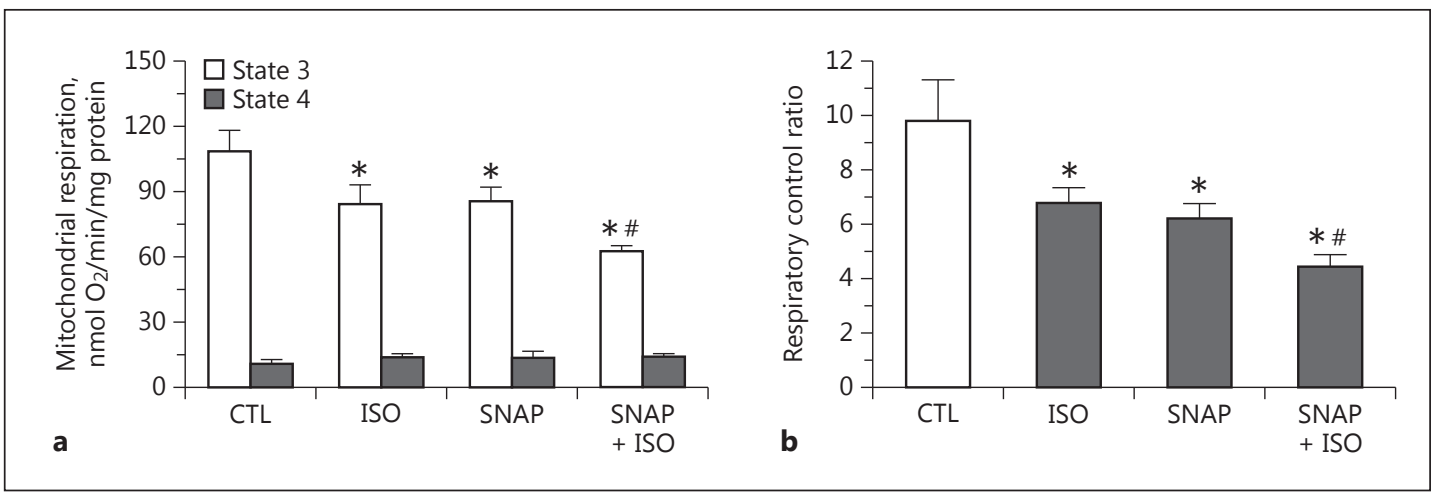

Fig. 5. Summarized data of the effects of the NO donor SNAP (1 $\mu \mathrm{M}$, applied in state 3$)$ on mitochondrial respiration (a) and respiratory control ratio (b) with or without isoflurane (applied in state 2) in the presence of the complex I substrates glutamate and malate. ${ }^{*} p<0.05$ compared to control group. ${ }^{\#} p<0.05$ compared to isoflurane group. CTL, control group; ISO, isoflurane.

(Fig. 4). L-NIO given in state 3 with or without isoflurane treatment did not have any significant effect on mitochondrial respiration when compared to the control group or the isoflurane-only group (data not shown).

\section{Role of Exogenous NO in the Effect of Isoflurane}

To observe the effect of exogenous NO on mitochondrial respiration, the NO donor SNAP was added before (Fig. 1b) or after ADP-initiated state 3 respiration (Fig. 1c). SNAP alone added before substrate administration (state 2) did not alter mitochondrial respiration and did not affect isoflurane-induced respiration changes (data not shown). However, SNAP administered in state 3 attenuated state 3 respiration compared to in the control (Fig. 5a; 86.2 \pm 6.3 vs. $108.8 \pm 9.3 \mathrm{nmol} O_{2} / \mathrm{min} / \mathrm{mg}$ protein, $p<0.05$ ). In the presence of isoflurane, SNAP further enhanced the attenuation of state 3 respiration ( $78 \pm 11$ vs. $61 \pm 5 \%, \mathrm{nmol} \mathrm{O}_{2} / \mathrm{min} /$ mg protein, $p<0.05$ ), but did not alter the increase in state 4 respiration by isoflurane. The decrease of RCR with SNAP in the presence of isoflurane was greater than in the isoflurane group or the SNAP-only group (Fig. $5 \mathrm{~b}$ ). 
Xu et al.: Is NO Involved in the Effect of Mitochondrial Complex I-Linked Respiration by Isoflurane?

\section{Discussion}

A better understanding of how isoflurane and other volatile anesthetics mediate cardioprotection against ischemia and reperfusion injury could have clinical implications. The cardioprotective effects of volatile anesthetics, including isoflurane, have, in part, been attributed to the direct inhibition of mitochondrial electron transport system complexes, and altered mitochondrial bioenergetics in hearts [5-8]. Although an attenuation of mitochondrial state 3 respiration by volatile anesthetics and the role of ROS have been reported [5], the effect of isoflurane administered in different states of mitochondrial respiration and the role of $\mathrm{NO}$ in this process have not been investigated.

This study shows that (1) isoflurane administrated in state 2 increased state 2 respiration and decreased state 3 respiration, (2) the decrease in state 3 respiration was greater when isoflurane was applied in state 3 than in state 2, (3) the NOS inhibitor L-NIO did not alter the effect of isoflurane on respiration, and (4) the NO donor SNAP administered during state 3 respiration enhanced the isoflurane-induced attenuation of state 3 respiration.

We found that isoflurane induced an increase in state 2 respiration and a decrease in RCR compared to the control group. These results suggest that isoflurane may cause a small proton leak through the inner mitochondrial membrane, and partially uncouple respiration, resulting in increased respiration. It has been reported that isoflurane attenuated the magnitude and increased the duration of state $3 \mathrm{NADH}$ oxidation. These effects are likely due to restricted proton pumping by complex I causing a slowed and prolonged proton entry into complex $\mathrm{V}$ after adding ADP [7].

Ischemic preconditioning also induces a mild proton leak that is involved in the mechanism of cardioprotection [19]. This uncoupling effect of isoflurane during state 2 or state 4 respiration may contribute to the mechanism of APC-induced cardioprotection. Uncoupling decreases proton driving force $\left(\Delta \mu_{\mathrm{H}+}\right)$ to facilitate the electron flow and reduce the toxic increase of superoxide production [20]. It has been confirmed that isoflurane preconditioning elicits partial mitochondrial uncoupling, with preserved respiration and ATP synthesis and a reduced mitochondrial $\mathrm{Ca}^{2+}$ uptake [6].

Isoflurane administered in state 2 attenuated state 3 respiration and decreased RCR during the oxidation of the complex I-linked substrates glutamate and malate. This effect of isoflurane is consistent with the findings of the studies by Agarwal et al. [7] and Riess et al. [5]. Riess et al. [5] found that sevoflurane reduced state 3 respiration when applied in state 2 . To observe the direct effect of isoflurane on state 3 respiration independent of its effect on state 2 respiration, we added isoflurane in state 3 . We observed that the attenuation of respiration by isoflurane was greater when it was added in state 3 than when it was added in state 2 .

Although the mechanism causing this different effect of isoflurane on different respiratory states is not known, isoflurane may inhibit enzyme activity in the respiring state (state 3 ) more effectively than in the quiescent state (state 2). Cytochrome c oxidase (complex IV) is known as the terminal enzyme in the mitochondrial respiratory chain. It reduces dioxygen to water, using reducing equivalents supplied by ferrocytochrome c $[16,21]$. It limits the respiration rate in the different respiring states. The control coefficient of cytochrome $c$ oxidase over mitochondrial respiration is higher in state 3 than in state 4 [22]. Therefore, the inhibition of isoflurane on cytochrome c oxidase could be more significant when it is applied in state 3. Cytochrome c oxidase is more active during ADP-initiated phosphorylation. In addition, the increase of state 2 respiration by isoflurane may also contribute to the further decrease in state 3 respiration than when isoflurane was administered in state 3.

Recent studies have proposed that mitochondria contain NOS and can produce significant amounts of NO to regulate respiration $[9,19]$. Therefore, NO may modulate the effects 
Xu et al.: Is NO Involved in the Effect of Mitochondrial Complex I-Linked Respiration by Isoflurane?

of volatile anesthetics on mitochondrial respiration. NO acts as a multisite inhibitor of the mitochondrial ETC. The most sensitive and widely studied target for NO in mitochondria is the terminal enzyme of the ETC, cytochrome c oxidase $[9,23,24]$. NO competes with $\mathrm{O}_{2}$ at the binuclear $\mathrm{Cu}_{\mathrm{B}} /$ cytochrome $\mathrm{a}_{3}$ site. This competition with $\mathrm{O}_{2}$ results in the inhibition of the enzyme, suggesting that $\mathrm{NO}$ is an important physiological regulator of mitochondrial oxidative phosphorylation $[9,23,24]$. Reports $[25,26]$ that the presence of the mitochondrial NOS isoenzyme is a constitutive protein in the mitochondrial inner membrane support a potential physiological role for NO in mitochondrial respiration. Mitochondrial NOS activity has been shown to be susceptible to pharmacological regulation and metabolic states [27]. Volatile anesthetics may activate mitochondrial NOS to produce NO, which leads to the isofluraneinduced alteration of respiration. To test this hypothesis, the NOS inhibitor L-NIO was given before or after the administration of isoflurane. Our results showed that L-NIO did not affect mitochondrial respiration and did not alter the effect of isoflurane on mitochondrial respiration. This suggests that the effect of isoflurane on mitochondrial respiration is independent of endogenous mitochondrial NO generation.

In isolated mitochondria from cardiac myocytes, exogenous NO inhibited respiration and reduced the NAD(P)H redox state [28]. NO is able to inhibit the ETC, mainly at complex IV, regulating oxygen consumption and ATP generation [29]. The respiratory chain is inhibited by NO, either supplied exogenously or produced endogenously via NOS activation. The inhibition of respiration is reversible, although its dependence on concentration and time of exposure to NO is not clear [30]. We also tested whether exogenous NO modulates mitochondrial respiration in the presence of isoflurane. When the NO donor SNAP was added in state 3 respiration, we found that it attenuated state 3 respiration, consistent with other studies $[14,31]$. The results also showed that the attenuation of state 3 respiration by isoflurane was enhanced by presence of SNAP. This suggests that extramitochondrial NO attenuates state 3 respiration and has a synergistic action with isoflurane on mitochondrial respiration. Borutaite and Brown [32] found that the reversible inactivation of mitochondrial enzymes by SNAP in isolated heart mitochondria resulted in a significant increase in $\mathrm{H}_{2} \mathrm{O}_{2}$ production. Therefore, SNAP may increase $\mathrm{H}_{2} \mathrm{O}_{2}$ production, which may further inhibit complex I, potentially contributing to the attenuation of state 3 respiration. It has been reported that $\mathrm{H}_{2} \mathrm{O}_{2}$ inhibits oxygen consumption in state 3 , and further increases ROS production with complex I substrates. It does not associate with complex II substrate [33]. In the rat brain mitochondria, the NO substrate L-arginine was shown to decrease state 3 respiration when administered after ADP-initiated state 3 respiration [34]. It has been confirmed that, under physiological conditions, respiration is more sensitive to NO in state 3 than in state 4 , due to there being greater control over respiration by cytochrome c oxidase in state 3 [16]. Taking previous studies into account, our finding of an enhancing effect of SNAP on the isoflurane-induced attenuation of state 3 respiration suggests that exogenous NO is involved in the modulation of mitochondrial respiration, and that isoflurane enhanced the inhibition of mitochondrial respiration in the presence of exogenous NO.

One limitation of our study is that the production of NO and ROS was not measured. The effects of endogenous NO on mitochondrial respiration with or without isoflurane need to be clarified. A study showed that mitochondrial $\mathrm{K}_{\mathrm{ATP}}$ channel opening increased ROS production in isolated heart and liver mitochondria. The location of ROS generation is in complex I of the ETC [35]. Volatile anesthetics could increase the formation of ROS at complex I and/or complex III of the ETC by the reversible attenuation of mitochondrial electron transport [4]. The ROS generation then affects the mitochondrial respiration by acting on downstream effectors, including mitochondrial $\mathrm{K}_{\mathrm{ATP}}$ channels that are normally closed at physiologic ATP levels [36]. We cannot exclude the relative contributions of $\mathrm{K}_{\mathrm{ATP}}$ channel or other potassium channel opening in the isoflurane-induced effect on different mitochondrial respiration 
states. In addition, our in vitro experiments were generally carried out at atmospheric $\mathrm{O}_{2}$ concentrations. This gives an $\mathrm{O}_{2}$ concentration of about 150-200 $\mu \mathrm{M}$ in the chamber, which is above the concentrations that cells are exposed to in vivo. Whether $\mathrm{O}_{2}$ limitation of mitochondrial respiration occurs in vivo over the range of $\mathrm{O}_{2}(1-20 \mu \mathrm{M})$ remains to be determined [16]. Therefore, the role of exogenous or endogenous NO in the effect of isoflurane on mitochondrial respiration may depend on $\mathrm{O}_{2}$ tension.

\section{Conclusion}

Isoflurane has different effects on different states of mitochondrial respiration during the oxidation of the complex I substrates glutamate and malate. The uncoupling effect during state 2 respiration and the attenuation of state 3 respiration by isoflurane may contribute to the mechanism of APC-induced cardioprotection. Endogenous mitochondrial NO is not involved in the effect of isoflurane on mitochondrial respiration.

\section{Disclosure Statement}

The authors have no conflicts of interest to disclose.

\section{Sources of Funding}

This work was supported by grants No. SS201756 (to Dr. An) from the Science and Technology Development Plan of Suzhou City; No. BK20141187 (to Dr. Wang) from the Natural Science Foundation of Jiangsu Province, and No SYS201473 (to Dr. Qiao) from the Science and Technology Development Plan of Suzhou City, China. Dr. Wang also received support from the Project of Gusu Health Key Talent (SS201613).

\section{References}

1 An J, Varadarajan SG, Novalija E, Stowe DF: Ischemic and anesthetic preconditioning reduces cytosolic (Ca2+) and improves $\mathrm{Ca}(2+)$ responses in intact hearts. Am J Physiol Heart Circ Physiol 2001;281:H1508-H1523.

2 Wojtovich AP, Smith CO, Urciuoli WR, Wang YT, Xia XM, et al: Cardiac Slo2.1 is required for volatile anesthetic stimulation of K+ transport and anesthetic preconditioning. Anesthesiology 2016;124:1065-1076.

3 Tian Y, Li H, Liu P, Xu JM, Irwin MG, et al: Captopril pretreatment produces an additive cardioprotection to isoflurane preconditioning in attenuating myocardial ischemia reperfusion injury in rabbits and in humans. Mediators Inflamm 2015;2015:819232.

4 Ludwig LM, Tanaka K, Eells JT, Weihrauch D, Pagel PS, et al: Preconditioning by isoflurane is mediated by reactive oxygen species generated from mitochondrial electron transport chain complex III. Anesth Analg 2004;99:1308-1315.

5 Riess ML, Eells JT, Kevin LG, Camara AK, Henry MM, et al: Attenuation of mitochondrial respiration by sevoflurane in isolated cardiac mitochondria is mediated in part by reactive oxygen species. Anesthesiology 2014; 100:498-505.

6 Ljubkovic M, Mio Y, Marinovic J, Stadnicka A, Warltier DC, et al: Isoflurane preconditioning uncouples mitochondria and protects against hypoxia-reoxygenation. Am J Physiol Cell Physiol 2007;292:C1583-C1590.

7 Agarwal B, Dash RK, Stowe DF, Bosnjak ZJ, Camara AK: Isoflurane modulates cardiac mitochondrial bioenergetics by selectively attenuating respiratory complexes. Biochim Biophys Acta 2014;1837:354-365.

8 Hanley PJ, Ray J, Brandt U, Daut J: Halothane, isoflurane and sevoflurane inhibit NADH:ubiquinone oxidoreductase (complex I) of cardiac mitochondria. J Physiol 2002;544:687-693.

9 Li H, Lang XE: Protein kinase C signaling pathway involvement in cardioprotection during isoflurane pretreatment. Mol Med Rep 2015;11:2683-2688.

10 Swyers T, Redford D, Larson DF: Volatile anesthetic-induced preconditioning. Perfusion 2014;29:10-15.

11 Bolli R: Cardioprotective function of inducible nitric oxide synthase and role of nitric oxide in myocardial ischemia and preconditioning: an overview of a decade of research. J Mol Cell Cardiol 2001;33:1897-1918. 
12 Chiari PC, Bienengraeber MW, Weihrauch D, Krolikowski JG, Kersten JR, et al: Role of endothelial nitric oxide synthase as a trigger and mediator of isoflurane-induced delayed preconditioning in rabbit myocardium. Anesthesiology 2005;103:74-83.

13 Ge ZD, Pravdic D, Bienengraeber M, Pratt PF Jr, Auchampach JA, et al: Isoflurane postconditioning protects against reperfusion injury by preventing mitochondrial permeability transition by an endothelial nitric oxide synthase-dependent mechanism. Anesthesiology 2010;112:73-85.

14 Brown GC: Nitric oxide and mitochondrial respiration. Biochim Biophys Acta 1999;1411:351-369.

15 Shiva S, Oh JY, Landar AL, Ulasova E, Venkatraman A, et al: Nitroxia: the pathological consequence of dysfunction in the nitric oxide-cytochrome c oxidase signaling pathway. Free Radic Biol Med 2005;38:297-306.

16 Brookes PS, Kraus DW, Shiva S, Doeller JE, Barone MC, et al: Control of mitochondrial respiration by NO*, effects of low oxygen and respiratory state. J Biol Chem 2003;278:31603-31609.

17 Saborido A, Soblechero L, Megias A: Isolated respiring heart mitochondria release reactive oxygen species in states 4 and 3. Free Radic Res 2005;39:921-931.

18 Paital B, Chainy GB: Effects of temperature on complexes I and II mediated respiration, ROS generation and oxidative stress status in isolated gill mitochondria of the mud crab Scylla serrata. J Therm Biol 2014;41:104111.

19 Nadtochiy SM, Tompkins AJ, Brookes PS: Different mechanisms of mitochondrial proton leak in ischaemia/ reperfusion injury and preconditioning: implications for pathology and cardioprotection. Biochem J 2006; 395:611-618.

20 Lenaz G: The mitochondrial production of reactive oxygen species: mechanisms and implications in human pathology. IUBMB Life 2001;52:159-164.

21 Zaslavsky D, Gennis RB: Proton pumping by cytochrome oxidase: progress, problems and postulates. Biochim Biophys Acta 2000;1458:164-179.

22 Groen AK, Wanders RJ, Westerhoff HV, van der Meer R, Tager JM: Quantification of the contribution of various steps to the control of mitochondrial respiration. J Biol Chem 1982;257:2754-2757.

23 Pannala VR, Camara AK, Dash RK: Modeling the detailed kinetics of mitochondrial cytochrome c oxidase: catalytic mechanism and nitric oxide inhibition. J Appl Physiol 2016;121:1196-1207.

24 Sarti P, Arese M, Forte E, Giuffre A, Mastronicola D: Mitochondria and nitric oxide: chemistry and pathophysiology. Adv Exp Med Biol 2012;942:75-92.

25 Zaobornyj T, Ghafourifar P: Strategic localization of heart mitochondrial NOS: a review of the evidence. Am J Physiol Heart Circ Physiol 2012;303:H1283-H1293.

26 Kato K, Giulivi C: Critical overview of mitochondrial nitric-oxide synthase. Front Biosci 2006;11:2725-2738.

27 Zaobornyj T, Valdez LB: Heart mitochondrial nitric oxide synthase: a strategic enzyme in the regulation of cellular bioenergetics. Vitam Horm 2014;96:29-58.

28 Kohlhaas M, Nickel AG, Bergem S, Casadei B, Laufs U, et al: Endogenous nitric oxide formation in cardiac myocytes does not control respiration during beta-adrenergic stimulation. J Physiol 2017;595:3781-3798.

29 Tengan $\mathrm{CH}$, Moraes CT: NO control of mitochondrial function in normal and transformed cells. Biochim Biophys Acta 2017;1858:573-581.

30 Sarti P, Arese M, Bacchi A, Barone MC, Forte E, et al: Nitric oxide and mitochondrial complex IV. IUBMB Life 2003;55:605-611.

31 Cleeter MW, Cooper JM, Darley-Usmar VM, Moncada S, Schapira AH: Reversible inhibition of cytochrome c oxidase, the terminal enzyme of the mitochondrial respiratory chain, by nitric oxide. Implications for neurodegenerative diseases. FEBS Lett 1994;345:50-54.

32 Borutaite V, Brown GC: S-nitrosothiol inhibition of mitochondrial complex I causes a reversible increase in mitochondrial hydrogen peroxide production. Biochim Biophys Acta 2006;1757:562-566.

33 Sanz A, Caro P, Gomez J, Barja G: Testing the vicious cycle theory of mitochondrial ROS production: effects of $\mathrm{H} 2 \mathrm{O} 2$ and cumene hydroperoxide treatment on heart mitochondria. J Bioenerg Biomembr 2006;38:121-127.

34 Lores-Arnaiz S, D’Amico G, Czerniczyniec A, Bustamante J, Boveris A: Brain mitochondrial nitric oxide synthase: in vitro and in vivo inhibition by chlorpromazine. Arch Biochem Biophys 2004;430:170-177.

35 Andrukhiv A, Costa AD, West IC, Garlid KD: Opening mitoKATP increases superoxide generation from complex I of the electron transport chain. Am J Physiol Heart Circ Physiol 2006;291:H2067-H2074.

36 Zaugg M, Lucchinetti E, Spahn DR, Pasch T, Schaub MC: Volatile anesthetics mimic cardiac preconditioning by priming the activation of mitochondrial K(ATP) channels via multiple signaling pathways. Anesthesiology 2002;97:4-14. 